\title{
La organización de las escuelas secundarias del estado de Chihuahua, en el marco de la reforma educativa
}

\section{David Manuel Arzola Franco \\ Profesor investigador del \\ Centro de Investigación y Docencia}

\section{Resumen}

1 1 presente artículo se deriva de un proyecto de investigación apoyado por el Fondo Mixto CONACYT-Gobierno del Estado de Chihuahua, cuyo propósito consiste en indagar sobre la visión que tienen los actores involucrados en la Reforma de la Educación Secundaria (RS), con respecto a los procesos de transformación que se pretenden operar en el ámbito organizativo.

El trabajo se llevó a cabo a través de la aplicación de una encuesta, a una muestra representativa de docentes, directivos, alumnos y padres/madres de familia, de escuelas secundarias, generales y técnicas, del estado de Chihuahua.

El análisis está centrado en algunos aspec- tos relacionados con la organización de los centros educativos: el funcionamiento institucional, los procesos comunicativos, las acciones de orientación y tutoría, el trabajo colaborativo; la información sobre la dinámica para la implementación de la Reforma de la Educación Secundaria (RS), y las perspectivas de los sujetos con respecto al manejo de los indicadores educativos a nivel institucional.

Los datos recabados ponen de relieve que la RS se limita al cambio del plan y los programas de estudio; no se advierten transformaciones en la estructura organizativa de las escuelas de este nivel: formas de gobierno, aspectos normativos, procesos de comunicación y participación, toma de decisiones, etc.

Palabras clave: organización, gestión, dinámica, colectivo, funcionamiento. 


\section{Introducción}

Con la modificación de los artículos $3^{\circ} \mathrm{y}$ 31 de la Constitución Política de los Estados Unidos Mexicanos, y la emisión de la Ley General de Educación en el año de 1993, la secundaria se incorpora al sistema de educación básica y adquiere un carácter obligatorio.

Con esa reforma, la educación secundaria es considerada como la etapa final o de cierre de la educación básica, lo cual demandó en su momento, además de una nueva propuesta curricular, una estrecha vinculación con el nivel de educación primaria, así como de acciones tendientes al mejoramiento de la gestión escolar, equipamiento de las escuelas y la actualización de los maestros (SEP, 2007). No obstante, para el 2005, doce años después de iniciado este proceso, la Secretaría de Educación Pública acepta que "en la práctica no se ha logrado una efectiva vinculación con los niveles previos de la educación básica". Es decir, la "pretensión de configurar un solo ciclo formativo con propósitos comunes, prácticas pedagógicas congruentes, así como formas de organización y de relación interna que contribuyan al desarrollo integral de los estudiantes" (SEP, 2005: 1), no había rendido los frutos esperados.

Ante este panorama, el 26 de mayo de 2006, por acuerdo secretarial publicado en el Diario Oficial de la Federación se inicia el proceso de Reforma de la Educación Secundaria, con la que se pretende generar amplios cambios en el quehacer educativo: el currículo, organización, infraestructura y equipamiento; estrategias para la formación, capacitación y actuali- zación de los profesores; así como la generación de nuevos mecanismos de información, comunicación y participación; elementos necesarios para que los colectivos puedan operar en el marco de esta nueva realidad.

Con este escenario a la vista surge el interés por desarrollar un proyecto de investigación con el propósito de conocer la visión que tienen los actores involucrados en la RS, con respecto a los procesos de transformación que pretenden operar en el ámbito organizativo, trabajo que se llevó a cabo en las escuelas secundarias -generales y técnicas- del Estado de Chihuahua.

Se trata de un estudio descriptivo que se realizó a partir del diseño de una encuesta aplicada a una muestra de 74 escuelas secundarias federalizadas -generales y técnicas-, seleccionadas al azar. En total se aplicaron 1117 instrumentos para docentes, 104 para directivos, 992 a padres y madres de familia y 1118 para alumnos y alumnas.

Para obtener la muestra, de los diferentes sujetos se utilizó el programa sTAST con el que se realizaron los cálculos en función de la población con que se contaba de acuerdo con la información proporcionada por el Departamento de Estadística de los Servicios Educativos del Estado de Chihuahua. De acuerdo con este programa se requerían entre 300 y 400 sujetos para tener una muestra representativa estatal, sin embargo nuestros números se elevaron considerablemente porque el equipo tomó la decisión de tomar muestras representativas por región: centro, norte, sur y sierra. 
Con respecto al procedimiento para elegir las escuelas, se utilizó la secuencia de números aleatorios que proporciona el mismo programa STAST. En el caso de docentes y directivos se encuestó al total de la población de las escuelas seleccionadas, mientras que en el caso de alumnos y padres de familia se trabajó con muestras escogidas de manera aleatoria.

El instrumento para docentes se compone de 52 reactivos -con cinco opciones de respuesta: totalmente de acuerdo, de acuerdo, neutral, en desacuerdo y totalmente en desacuerdo- y siete preguntas abiertas. Los instrumentos para directivos y padres/madres de familia se componen de 56 y 30 reactivos respectivamente - con cinco opciones de respuesta- y seis y tres preguntas abiertas respectivamente. Finalmente, el instrumento para alumnos se compone de 30 reactivos con las mismas cinco opciones de respuesta, en este caso no se incluyeron preguntas abiertas.

El procesamiento de la información se realizó mediante un análisis de frecuencias con el apoyo del programa estadístico SPSS (Versión 15.0).

\section{Análisis de resultados}

\section{Funcionamiento institucional}

La transformación curricular tuvo una implicación importante en la distribución de las cargas horarias, de acuerdo con los documentos oficiales, "se redujo el número de asignaturas por grado a fin de favorecer la comunicación entre docentes y estudian- tes, así como de propiciar la integración de las asignaturas e incidir positivamente en los aprendizajes de los alumnos" (SEP, 2006a:26), esta circunstancia trajo consigo la necesidad de hacer ajustes a nivel institucional. De acuerdo con los datos obtenidos, el $80 \%$ de los directivos opinan que el perfil profesional de los docentes se corresponde con las materias que imparten, mientras que el $92 \%$ de profesores están de acuerdo o totalmente de acuerdo con esta afirmación. No obstante $57 \%$ de directivos y el $31 \%$ de docentes opinan que los profesores están atendiendo asignaturas con las que no habían trabajado anteriormente (ver gráfica 1). Esta circunstancia resulta normal ante un proceso de renovación del currículo, sin embargo para las instituciones significa un esfuerzo considerable y diferenciado para responder a las necesidades de capacitación y actualización docente, ya que estos profesores en particular tienen que atender de manera simultánea el manejo del nuevo plan y programas de estudio, así como actualizarse en relación con el conocimiento disciplinar de las nuevas materias que les fueron asignadas.
Gráfica 1. Los docentes imparten materias con las que no habían trabajado antes

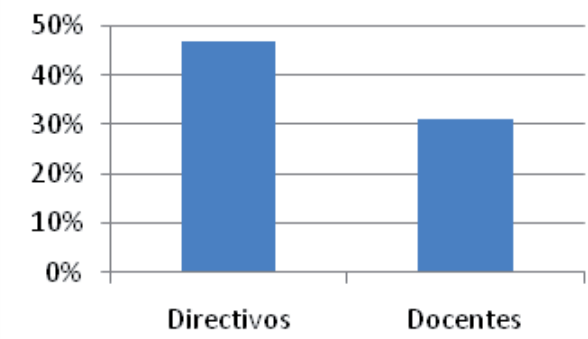

- De acuerdo o totalmente de acuerdo 
Por otra parte, y de manera proporcional, la percepción de cambio se destaca más en los docentes que en los directivos (ver gráfica 2), lo que pone de manifiesto que la parte sustancial de la Reforma está centrada en los programas que son operados directamente por los profesores. Lo anterior sugiere que el resto de las propuestas que acompañan a los cambios curriculares, como en el caso de las "nuevas formas de organización y gestión", tienen un carácter epidérmico, es decir se encuentran expresadas a nivel discursivo pero no se traducen en estrategias y acciones a nivel de los colectivos escolares. En concreto, no se encuentran evidencias de cambios importantes en cuanto a la estructura organizativa de las escuelas a partir de la generalización de la Reforma, los datos que se presentan a continuación nos permiten apoyar este supuesto.

De acuerdo con la información recabada, solamente poco más de la mitad de los directivos (54.4\%) están de acuerdo en que a partir de la Reforma se ha modificado su carga de trabajo, mientras que este porcentaje se eleva al $63.5 \%$ en el caso de los docentes. Cuando se les preguntó si esas modificaciones les han facilitado el trabajo, solamente el $40 \%$ de los directivos y el 50\% de los docentes están de acuerdo. Estas opiniones son muy cercanas a los porcentajes que presentan los alumnos y los padres/madres de familia cuando se les pregunta si creen que los docentes tienen de-
Gráfica 2. A partir de la reforma ha habido cambios a nivel institucionl

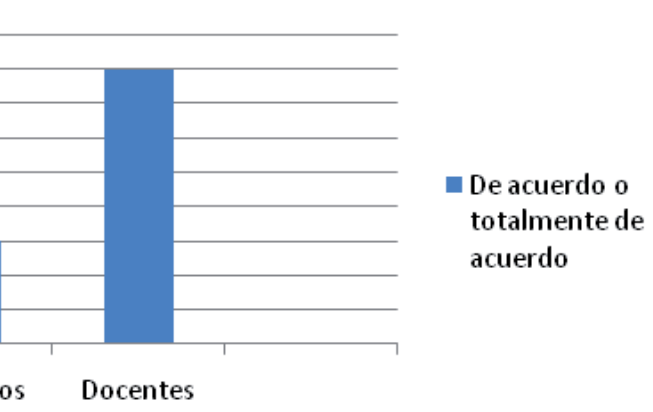

masiado trabajo: el 52\% de los alumnos y el $46 \%$ de los padres están de acuerdo con la afirmación.

Una de las exigencias para la educación del siglo XXI consiste en enfocar la función de la escuela en el aprendizaje de los alumnos (Namo de Melo: 1998), esto implica que las funciones administrativas tengan que supeditarse a las de carácter pedagógico, al respecto el $61 \%$ de los docentes consideran que a partir de la reforma se da más importancia a los aspectos pedagógicos, sin embargo solamente $31 \%$ de directivos y $25 \%$ de docentes están de acuerdo o totalmente de acuerdo en que a partir de la reforma se ha simplificado la carga administrativa y solo el $50 \%$ de los directivos consideran que a partir de la reforma se modificó la normatividad interna de las escuelas. Estos aspectos son cruciales para impulsar un cambio de fondo en las instituciones, sin embargo la información sugiere que más allá del cambio en los programas no se han impulsado ningún otro tipo de transformaciones, sobre todo en el ámbito administrativo: 
Una paradoja que la mayoría de los políticos y administradores todavía no han sabido apreciar en su totalidad se refiere a que la demanda de papeleo que tiene como meta mejorar la rendición de cuentas... en realidad afecta negativamente a la productividad, en la medida en que resta tiempo a las funciones centrales de la escuela (DarlingHammond, 2002: 85).

Además del cambio curricular resulta evidente que las escuelas requieren de cambios profundos en lo cultural, así como en su estructura y organización, de tal manera que se cuente con la flexibilidad necesaria para que se puedan impulsar acciones innovadoras, estas condiciones aparentemente no están acompañando al proceso de generalización de la Reforma. En el caso del cambio normativoorganizativo, la intención de impulsar procesos de transformación está presente a nivel del discurso en los documentos generados por la RS, sin embargo los datos anteriores sugieren que no hay estrategias encaminadas en esa dirección, es decir las escuelas aparentemente están operando de la manera en que lo han hecho tradicionalmente. Por ejemplo, a nivel estatal no se ha presentado ninguna iniciativa para elaborar una normatividad para la organización y el funcionamiento de los centros de educación secundaria, y por tanto ésta "continúa rigiéndose por normas que datan de los años 80 y que tuvieron como marco la Ley Federal de Educación de 1973, previa al ANMEB y a la obligatoriedad de la educación secundaria" (SEP, 2005a: 18), normas que evi- dentemente resultan obsoletas para el marco de exigencias en que se mueve la educación secundaria en el momento actual.

Sin embargo estos cambios de carácter formal no garantizan por sí solos la transformación de las instituciones, se requiere de un impulso desde los propios centros educativos, es necesario pues, una transformación cultural, tal como lo expresa Justa Ezpeleta:

Los estudios del cambio educativo han coincidido en señalar que la innovación en las prácticas docentes tiene mayor posibilidad de éxito y arraigo cuando han surgido de las propias escuelas... En la situación opuesta, es decir cuando el cambio es inducido y se propone afectar al conjunto de un nivel... lo que se encuentra consistentemente es la tendencia a resistirlo por parte de los actores y de las estructuras escolares (Ezpeleta, 2004: 404).

En este sentido el doble reto que enfrenta la educación secundaria de cambiar no solo en lo normativo-organizativo sino de manera simultánea transformarse en lo cultural, no implica otra cosa que aprender a lidiar con una realidad cuyo sello característico es el del cambio permanente, por ello "la transformación intencionada que es la educación debe sufrir continuas innovaciones a fin de ajustarse a contextos cambiantes y/o de mejorar la calidad de lo que hace. Si seguimos haciendo lo mismo, las cosas seguirán igual. No podremos mejorar nuestra educación si no innovamos" (Schmelkes, 2001). 


\section{Procesos comunicativos y clima institucional}

En este apartado se abordan las características del medio ambiente en el que se desarrolla el trabajo en las escuelas secundarias, "el clima institucional representa la personalidad de un centro, en cuanto es algo original y específico del mismo con un carácter relativamente permanente y estable en el tiempo, cuya evolución se realiza lentamente aunque se modifiquen las condiciones. El concepto de clima institucional tiene un carácter multidimensional y globalizador" (Rodríguez, 2004).

En relación con los procesos de interacción se detectó una brecha importante en las opiniones de los diferentes actores: el 88\% de los directivos y $58 \%$ de docentes consideran que la comunicación interna es ágil y efectiva. Por su parte, el $75 \%$ de los padres/madres de familia dicen que la escuela mantiene una buena comunicación con ellos. (Ver gráfica 3).

Gráfica No. 3
La mayoría de los profesores, el 70\%, señalan que los mecanismos de comunicación que comúnmente utilizan para contactar a padres y madres de familia son eficientes. En el mismo sentido el $95 \%$ de los padres/madres de familia comentan que los citan para darles a conocer los resultados de la evaluación de sus hijos, y el $92 \%$ dicen que reciben esta información de manera permanente. Mientras que el $80 \%$ de los alumnos consideran que los docentes los mantienen bien informados sobre los resultados del grupo.

Los datos anteriores nos hablan de un tipo de comunicación que fluye de manera adecuada entre la escuela y los padres de familia, sin embargo parece que ésta se refiere solamente a la información sobre los resultados en el aprovechamiento de los estudiantes, cuando se trata de información acerca de otros procesos que atañen a la vida institucional el porcentaje tiene un sensible declive: solo seis de cada diez de los padres/madres encuestados dicen que están bien informados sobre la manera en que

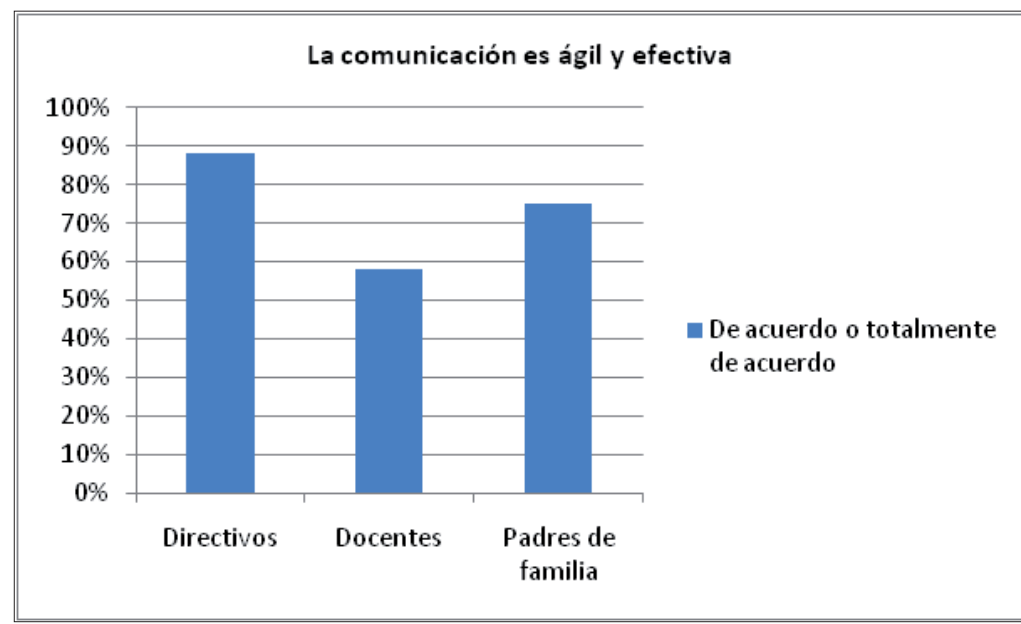
está organizada la escuela (ver gráfica 4 ).

Por otra parte, solo el $55 \%$ de alumnos dicen que los maestros tienen una buena comunicación con los padres, y los mantienen informados de lo que pasa en la escuela. Este aspecto es importante porque en los "procesos relacionados con la construcción del ambiente de 
aprendizaje en la escuela, se destaca... el papel protagónico y cada vez más activo de los alumnos, (y) la presencia de los padres como actores significativos dentro del sistema escolar" (LLECE, 2002: 11).

Gráfica No. 4

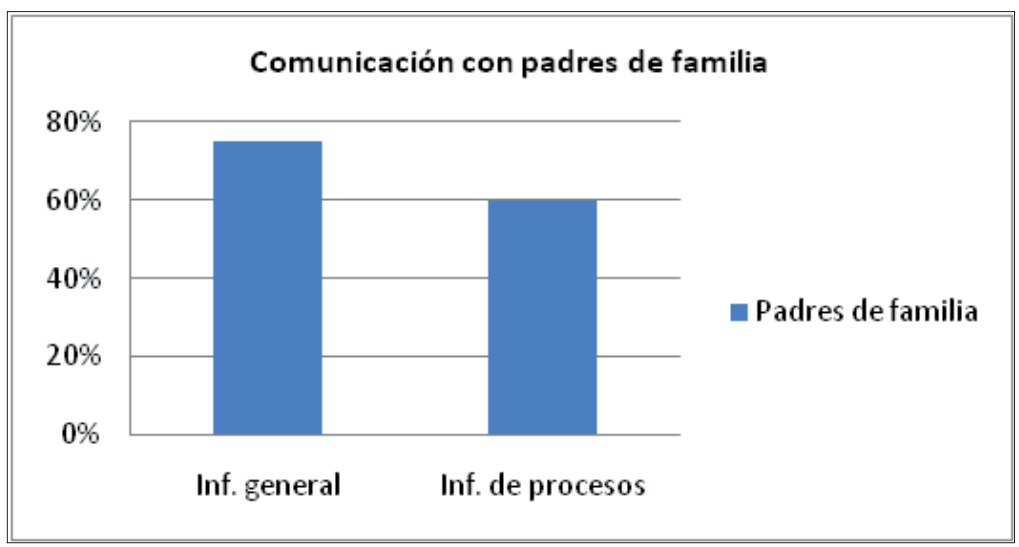

Con respecto a la comunicación hacia el exterior, el 60\% de los directivos están de acuerdo o totalmente de acuerdo en que a partir de la reforma se han implementado nuevos mecanismos de comunicación con las autoridades educativas, mientras que los docentes parecen advertir estos cambios en menor medida, solo el $42 \%$ están de acuerdo con esa afirmación.

En lo que atañe al clima institucional, encontramos que la tendencia en las opiniones es positiva ya que se encuentran proporciones cercanas o por encima del $70 \%$ en las que los informantes dicen estar de acuerdo o totalmente de acuerdo con las proposiciones del instrumento. No obstante también se encontraron algunas opiniones discrepantes:
Los docentes y directivos tienen distintas percepciones respecto al ambiente de trabajo, el $86 \%$ de los directivos consideran que se trabaja en un ambiente de respeto y solidaridad, mientras que solo un $65 \%$ de los docentes están de acuerdo con esta afirmación.

Por otra parte, el $73 \%$ de los directivos consideran que la dirección cuenta con mecanismos de consulta para conocer las inquietudes $y$ necesidades académicas del personal. El $69 \%$ de los docentes y el $70 \%$ de los padres por su parte señalan que los directivos/as escuchan y aceptan las sugerencias académicas que se les hacen, de igual manera el $79 \%$ de los padres y madres de familia dicen que los/as directivos escuchan con interés, cuando alguien se acerca a platicar con ellos. Y un $66 \%$ de los padres dicen que con frecuencia los directivos/as están presentes en las reuniones con padres y madres de familia. En cambio un porcentaje considerable de alumnos dicen que sus opiniones no son tomadas en cuenta y visualizan a la figura de los directivos como distante de la actividad en las aulas: solo el $57 \%$ están de acuerdo en que el cuerpo directivo toma en cuenta la opinión de las y los alumnos para mejorar la escuela y que el director se relaciona bien con la mayor parte de los alumnos. Además solo el $34 \%$ dice que con frecuencia los directivos están presentes en las exposiciones de los trabajos de los grupos. 
De acuerdo con los niveles de respuesta obtenidos en este aspecto, es importante destacar que los estudiantes ocupan una posición periférica en cuanto a la participación, aparentemente ésta no se visualiza como un recurso pedagógico crucial para la formación ciudadana, la responsabilidad y la inclusión de los niños y jóvenes en los procesos democráticos:

La participación se puede definir como un reconocimiento de lo que los niños son capaces de hacer e implica que los niños sean miembros de la sociedad, capaces de pensar y expresarse por sí mismos. Por tanto, no sólo tienen algo que decir sobre lo que afecta a su vida como persona, miembro de una familia y de una comunidad, sino que deben ser informados, consultados y escuchados en cuanto a todos esos aspectos (Hart, 2005).

El 63\% de los docentes dicen que las y los maestros disfrutan trabajar juntos en la escuela, lo que indica un porcentaje considerable de profesores que no están contentos con la manera en que se trabaja en la institución.

Por otra parte, el 70\% de los docentes señalan que el cuerpo directivo se relaciona bien con la mayor parte del personal, y el $81 \%$ expresan que los directivos se relacionan bien con los profesores/as. Mientras que el $85 \%$ de los padres dicen que las y los maestros se relacionan de manera cortés con los padres y madres de familia y el 79\% de los alumnos dicen que les gusta asistir a clases.

En lo que se refiere a las relaciones que se establecen entre los diferentes actores, se obtuvieron los siguientes datos: el $72 \%$ de los alumnos están de acuerdo en que los directivos/as procuran que los maestros/as, secretarias/os, conserjes, etc., los traten con amabilidad y respeto. En tanto que el $80 \%$ de los padres y madres de familia están de acuerdo en que las y los docentes están siempre dispuestos a ayudar les cuando lo necesitan. Además tanto los alumnos, $74 \%$, como los padres, $72 \%$, coinciden en que los profesores procuran que los estudiantes se interesen por conocer más sobre algunos temas importantes. Asimismo el $83 \%$ de los directivos y el $72 \%$ de los docentes dicen que en sus escuelas secundarias, se alienta y anima la autonomía de las y los docentes.

Con respecto a las responsabilidades del centro, la autoimagen del trabajo directivo no concuerda con la apreciación del resto de los actores: el 93\% de los directivos están de acuerdo en que ponen el ejemplo al ser los primeros en trabajar arduamente, en cambio solo el $62 \%$ de los docentes, el $60 \%$ de los alumnos, y el $66 \%$ de padres de familia apoyan esa afirmación.

En general podemos observar que las percepciones de los diferentes actores en lo que se refiere al clima institucional son positivas, no obstante también se encontraron algunas opiniones discrepantes: los docentes y directivos tienen distintas percepciones respecto a los procesos de comunicación y al ambiente de trabajo, la comunicación hacia el exterior no se percibe como algo que fluye de manera eficiente, la comunicación con los padres de 
familia resulta limitada, y finalmente un porcentaje considerable de alumnos no consideran que sus opiniones sean tomadas en cuenta y visualizan a la figura de los directivos como distante de la actividad en las aulas.

\section{Orientación y tutoría}

La orientación y tutoría es una de las novedades curriculares; y de acuerdo con los documentos oficiales,

el propósito (de este) Espacio Curricular es brindarles a los alumnos un acompañamiento en su integración a la vida académica de la escuela secundaria, promover en los adolescentes el reconocimiento de sus necesidades e intereses como estudiantes, a fin de coadyuvar en la conformación de un proyecto de vida, que les permita convivir y desenvolverse en la sociedad (SEP, 2006b).

Por ser de una actividad inédita resulta interesante analizar la experiencia acumulada en las instituciones al trabajar con esta modalidad.

Al tratarse de una actividad en la que los docentes tienen todavía poca experiencia, sus propósitos parecen no estar claramente definidos en los colectivos escolares: el 73\% de los directivos y $49 \%$ de los profesores consideran que los docentes tienen información suficiente sobre las responsabilidades y funciones de los tutores. Cuando se afirma que el tutor y los docentes están organizados para diseñar estrategias encaminadas a una mejor atención de alumnos: el $76 \%$ de los directivos y $51 \%$ de los docentes están de acuerdo, la diferencia entra las percepciones de ambos informantes es considerable.

En el mismo sentido el 64\% de los alumnos dicen que los profesores responsables de la asignatura de orientación y tutoría les han brindado apoyo cuando lo necesitan, el 60\% opinan que los docentes están bien organizados para poder atenderlos y solo el 50\% afirman que el tutor comunica a los profesores de otras asignaturas acerca de los problemas y necesidades de los alumnos. La opinión de padres/madres es la siguiente: $64 \%$ afirman que en la institución se ha informado de las actividades que realizan los tutores en los grupos.

En otras respuestas relacionadas con el mismo tema, el $68 \%$ de los alumnos señalan que el tutor informa al grupo sobre las actividades más importantes de la escuela, y el 64\% comentan que están satisfechos con la clase de orientación y tutoría.

\section{Trabajo colegiado}

En lo que se refiere a las modificaciones de los criterios que norman el trabajo colegiado $59 \%$ de los docentes consideran que se han operado algunos cambios. Este dato resulta alentador porque indica cierto nivel de movimiento en el ámbito institucional, mismo que en este momento puede ser muy incipiente pero que puede detonar procesos de cambio, sobre todo porque se trata de iniciativas que no necesariamente son impuestas sino que 
surgen de los colectivos. Al respecto Bolívar (1996) dice que "los tipos de cultura escolar que más apoyan la innovación son las basadas en la colaboración, tienen altas expectativas sobre los alumnos y profesores, exhibe un consenso sobre valores, apoyo del entorno, $y$ promueve profesores que puedan asumir una variedad de roles de liderazgo."

Por lo mismo, los colectivos escolares en el nivel de secundarias enfrentan el reto de generar dinámicas para integrar equipos de trabajo que permitan colegiar las decisiones de carácter académico. Lo anterior es una tarea compleja ya que

la integración de un equipo de trabajo requiere una finalidad muy clara, una red de personas sensibles con capacidad para expresarse sobre su quehacer de manera propositiva y reflexiva. Y además requiere de la claridad del equipo directivo para formar $y$ crear las condiciones necesarias para su desarrollo, desplegando, de esta manera, una verdadera pedagogía de la autonomía (Pozner, 2005: 88).

La mayoría de directivos (96\%) y un porcentaje considerable de docentes (69\%) señalan que en sus escuelas se promueven acciones enfocadas en el trabajo colaborativo. De igual manera, $90 \%$ de directivos y $71 \%$ de docentes, están de acuerdo, en que las reuniones académicas brindan elementos que apoyan el trabajo, es decir existe la convicción de que el trabajo colegiado es un recurso importante para los procesos de mejora. Pero solo el 51\% de directivos y el $29 \%$ de docentes consideran que cuentan con el tiempo suficiente para el desarrollo del mismo (ver gráfica 5), lo cual se convierte en uno de los puntos débiles del proceso ya que por una parte se detecta un alto nivel de exigencia para que los establecimientos impulsen el trabajo en equipos y la toma de decisiones consensuada y por otro lado no se dispone del tiempo indispensable para desarrollar esta tarea. Esto obliga a las escuelas a suspender labores o dedicar tiempo fuera de los horarios de clase para las reuniones académicas.

Gráfica No. 5

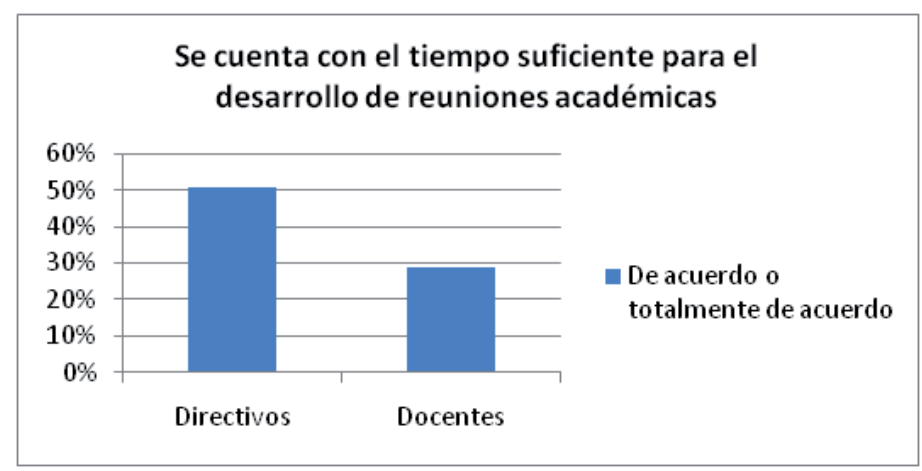

El gran reto para estas instituciones dada la precariedad en la que se desarrolla el trabajo colegiado consiste en evitar o superar dos problemas señalados por Bolívar (1993):

La cultura fragmentaria (o "balcanizada") (...) propia de escenarios en los que predominan los subgrupos, precisamente los mismos espacios en los que 
se verifica el aprendizaje profesional y que permanecen relativamente estables en el tiempo; existe, a su vez, una identificación por el subgrupo de pertenencia -con el que se comparten modos de pensar y enseñar- $y$ se asocia, frecuentemente, con la materia o el área (sobre todo en la educación secundaria); la organización escolar, por esto mismo, se estructura en función de los ámbitos disciplinares (materias, niveles, áreas, departamentos...).

La colegialidad artificial cuando las relaciones y el trabajo conjunto se impongan por procedimientos burocráticos o prescripciones administrativas; a la vez misma que el diseño del proyecto de centro y la elaboración curricular se verifiquen con escasa autonomía e insuficiente adaptación de marcos generales.

El reto que enfrentan los colectivos escolares es complejo porque el cambio curricular no está acompañado de las herramientas de carácter normativo para impulsar el trabajo académico en los centros educativos, por el contrario, la normatividad como ya se expresó anteriormente, resulta obsoleta y no está diseñada para dar respuesta a las necesidades actuales de las escuelas. Derivado de lo anterior, la organización escolar, con su enfoque burocrático-administrativo, tampoco parece haber sido alterada a partir de la RS, y por tradición no está diseñada para la generación de ambientes de aprendizaje y de autoaprendizaje retadores que impulsen la creatividad y el libre intercambio de ideas, la originalidad y el reto de enfrentar los procesos permanentes de cambio. "El trabajo docente en secundaria a diferencia de los otros niveles de la educación básica presenta características particulares que contribuyen a la fragmentación del cuerpo docente" (Sandoval, 2004: 152), la constante en las escuelas es entonces la lucha contra reloj para cumplir con los programas y por tanto el aislamiento más que la colegialidad parece ser el aspecto dominante de las prácticas.

\section{Manejo de indicadores educativos}

El $91 \%$ y $87 \%$ de directivos dicen que los indicadores educativos son un insumo para la toma de decisiones a nivel institucional y de la de zona escolar respectivamente. Asimismo el 93\% consideran que el análisis de los indicadores se traduce en estrategias concretas para la mejora institucional. Esta cifra es menor en el caso de los docentes: 70\% (ver gráfica 6).

Gráfica No. 6
El análisis de los indicadores educativos se traduce en estrategias para la mejora

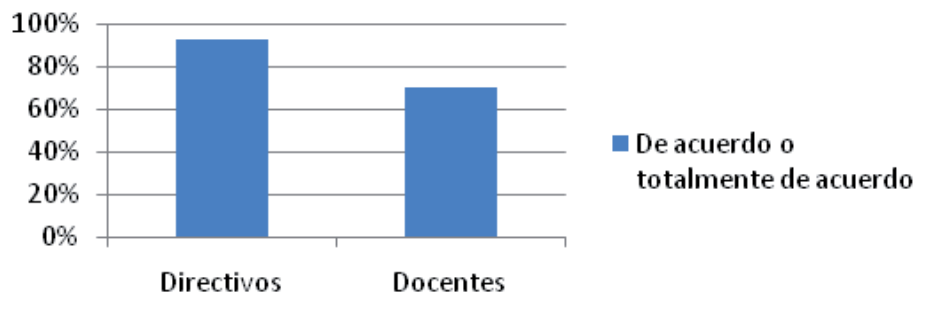


El $87 \%$ de directivos y $70 \%$ de docentes consideran que sus instituciones cuentan con estrategias definidas para atender los problemas de reprobación y bajo aprovechamiento (ver gráfica 7). Un 68\% de estudiantes comentan que si algún alumno reprueba o tiene bajas calificaciones, los maestros lo ayudan para mejorar su desempeño, el 78\% está de acuerdo en que los profesores o los directivos platican con los padres para que puedan ayudarlos cuando están reprobados. Las opiniones anteriores son compartidas por los padres de familia: $75 \%$ dicen que si algún alumno reprueba, los maestros lo ayudan para mejorar, y $83 \%$ está de acuerdo en que los profesores o los directivos platican con ellos para que puedan ayudar a sus hijos.

Gráfica No. 7

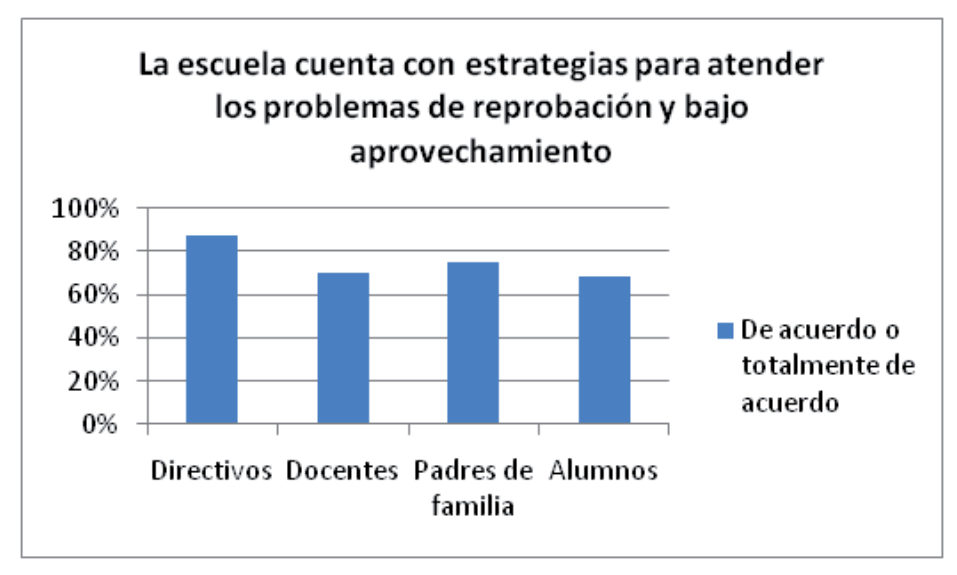

Sin embargo cuando se trata de atender el problema de la deserción las opiniones positivas descienden: el $64 \%$ de los directivos consideran que cuando se presenta el caso de alumnos desertores, la institución cuen- ta con estrategias para reincorporarlos, pero solo un $49 \%$ de los docentes comparten esta opinión. Se trata desde luego de un problema difícil de abordar considerando que la deserción escolar está ligado a variables de carácter socioeconómico que rebasan con mucho la capacidad de respuesta y los recursos con los que cuentan las escuelas. En un estudio sobre el rendimiento escolar realizado por Margarita Zorrilla "se muestra el poco peso del trabajo de la escuela en la compensación de las diferencias debidas a las características de origen, sociales, económicas y culturales existentes entre los alumnos" (Zorrilla, 2009).

\section{Conclusiones}

1. No se encuentran evidencias de cambio en cuanto a la estructura organizativa de las escuelas a partir de la generalización de la RS. Aunque evidentemente hay un cambio en el enfoque del plan y los programas de estudio, en lo organizativo se continúa laborando con el marco normativo-operativo pre-reforma. Esta condición resulta particularmente problemática porque pone en tela de juicio el carácter reformador de las estrategias desplegadas; el cambio del plan y los programas de estudio resulta insuficiente para hablar de una verdadera reforma.

2. Los aspectos relacionados con la implementación del nuevo currículo tales como la distribución de asignaturas y el trabajo de 
orientación y tutoría han causado cierto nivel de desconcierto y desajustes a nivel de las instituciones, situación que todavía no acaba de normalizarse, sin embargo estos problemas forman parte del proceso natural del reacomodo que desencadena cualquier movimiento de esta naturaleza.

3. Se detectan problemas en los procesos de comunicación entre los diferentes actores, por ello se requiere de nuevos mecanismos y estrategias que acerquen a los directivos, docentes, alumnos, padres y madres de familia al análisis en común de los problemas de las escuelas con la finalidad de buscar alternativas consensuadas.

4. El clima institucional presenta claroscuros, es importante resaltar que desde la visión externa los padres de familia perciben de manera positiva el trabajo de los docentes y directivos, sin embargo en lo interno es necesario atender cuestiones relacionadas con la información y toma de decisiones y centrar el interés en la atención a las necesidades de los estudiantes.

5. Existe un convencimiento pleno de los colectivos escolares acerca de los beneficios de trabajo colegiado, pero aceptan que no disponen de tiempo para desarrollarlo.

6. Resulta paradójico que por una parte se exija a las escuelas impulsar la vida académica y el trabajo colaborativo y por otra no se brinden espacios y herramientas de tipo legal o normativo para llevarlos a cabo. No obstante esta puede ser una de las alternativas para atender la diversidad de problemas educati- vos que se presentan en las escuelas; pero se requiere destinar tiempos institucionales para ese fin, así como el diseño de las estrategias adecuadas para dar sentido y dirección a la labor de los equipos para detonar los procesos de cambio desde el interior de los centros.

\section{Bibliografía}

Bolívar, A. (1996). Cultura escolar y cambio curricular. Bordón, 48 (2), 169-177. Universidad de Granada. Consultado el 20 de agosto de 2009 en: http://www.educacion.es/cide/ espanol/investigacion/rieme/documentos/files/bolivar3/ Bolivar3.pdf

Bolívar, A. (1993). Culturas profesionales en la enseñanza. Cuadernos de Pedagogía, 269, 68-72. Consultado el 20 de agosto de 2009 en: http://www.educacion.es/cide/espanol/investigacion/rieme/documentos/files/bolivar3/Bolivar3.pdf

Darling-Hammond, L. (2002). El derecho de aprender. México, D. F. Secretaría de Educación Pública.

Ezpeleta Moyano, J. (2004). Innovaciones educativas, reflexiones sobre los contextos en su implementación. Revista Mexicana de Investigación educativa, abril-junio 2004, Vol. 9, Núm. 21, pp 403-424.

Namo de Melo, G. (1998) Nuevas propuestas para la gestión educativa. Biblioteca del Normalista. México, SEP.

Pozner de Weinberg, P. (2005). El directivo como gestor de aprendizajes escolares, $5^{\text {a }}$. edición. Argentina, Aique grupo editor.

Rodríguez Garrán, N. (2004). El clima escolar, en Revista digital de "Investigación y Educación". Número 7, volumen 3, marzo de 2004. ISSN 1696-7208. Consultada en 10 de junio de 2009, en http://www.oposicionesprofesores.com/biblio/ docueduc/EL\%20\%20CLIMA\%20Y\%20LA\%20\%20CULTURA\%20INSTITUCIONAL\%20DE\%20LOS\%20CENTROS\%20DOCEN\%85.pdf

Schmelkes S.(2001). La investigación en la innovación educativa. Departamento de Investigaciones Educativas CINVESTAV. Consultado en febrero, 20, 2009 en http://bibliotecadigital.conevyt.org.mx/colecciones/redepja/Doc_1.pdf

SEP (2005a). Orientaciones generales para la elaboración de la normatividad en las entidades federativas. Del gobierno y funcionamiento de la Educación Secundaria. Consultado en: http://www.reformasecundaria.sep.gob.mx/doc/proc/ proconstru.pdf Fecha de consulta 7 de abril de 2008.

SEP (2005b). Propuesta curricular para la educación secundaria, proceso de construcción. Consultado en: http://www. reformasecundaria.sep.gob.mx/doc/proc/proconstru.pdf Fecha de consulta 7 de abril de 2008.

SEP (2006a). Plan de estudios 2006. Consultado en marzo, 5, 2009 en http://www.reformasecundaria.sep.gob.mx/doc/ 
REVISTA DE INVESTIGACIÓN EDUCATIVA DE LA REDIECH

programas/2006/planestudios2006.pdf

SEP (2006b). Espacio electrónico de orientación y tutoría. Consultado en febrero, 2, 2009 en http://www.reformasecundaria.sep.gob.mx/orientacion/index.htm.

SEP (2007). Plan de estudios de educación secundaria. Secretaría de Educación Pública, segunda edición, 2007.

Hart, R. (2005). Proyecto Educativo El libro de nuestra escuela. Una estrategia global para fomentar la participación de los alumnos de Educación Primaria. Revista gratuita editada por Editorial Planeta Grandes Publicaciones, septiembre 2005. Consultado el 23 de septiembre de 2009, en http:// www.librodenuestraescuela.com/Data/Pdf/Revista_LNE05CAST.pdf

Zorrilla Fierro, M. (2009). ¿Cuál es la aportación de la escuela secundaria mexicana en el rendimiento de los alumnos en Matemáticas y Español? Instituto Nacional para la Evaluación de la Educación. Vol. 11, Núm. 2, 2009. Consultado el 8 de octubre de 2009 en: http://redie.uabc.mx/vol11no2/ imprimir-contenido-zorrilla2.html 